\title{
DELETION OF MITOCHONDRIAL INORGANIC PYROPHOSPHATASE GENE EXTENDS LIFE SPAN IN HAPLOID YEAST (Saccharomyces cerevisiae)
}

\author{
Khandaker, A. M. and A. Koc ${ }^{1}$ \\ Department of Zoology, University of Dhaka, Dhaka-1000, Bangladesh; ${ }^{1}$ Genetics and Molecular \\ Biology department, Izmir Institute of Technology, Turkey
}

\begin{abstract}
Aging is a universal but poorly understood biological process and its underlying mechanisms are under intensive study. Mitochondria have a central role in the studies of aging hence they supply the majority of the organisms' energy requirement from biological fuels. However, the role of mitochondria in the aging process is more complicated than the proposed theories of aging. We addressed a question by asking whether deletion to mitochondrial metabolism genes can extend life span in haploid cell of Saccharomyces cerevisiae (yeast). In this study, strains derived from the yeast open reading frame (ORF) deletions were screened through replicative aging assay in order to identify the mitochondrial metabolism genes that increase life span. This has resulted in the isolation of a long living (22\% extended life span) mutant, ppa2 $\Delta$, which lack mitochondrial inorganic pyro phosphatase gene. The mitochondrial morphology of this long living mutant was analyzed by fluorescence microscopy. Compared to serpentine nature of wild type mitochondria, a different dynamics and distribution pattern was viewed, i.e. mitochondria were aggregated and formed colonies within the cytoplasm of this long lived cell. The aggregated mitochondrial mass was found to be intact from the young to old stage of life. Thus, this investigation reveals the longevity role of the mutant form of the gene PPA2 through an alteration of mitochondrial morphology.
\end{abstract}

Key words: Aging and longevity, ppa $2 \Delta$, altered mitochondrial morphology, replicative life span.

\section{INTRODUCTION}

Aging is a process that is defined as a progressive deterioration of biological functions after the organism has attained its maximal reproductive competence that leads to mortality (Farooqui and Farooqui 2008). The time-dependent accumulation of cellular damage is widely considered the general cause of aging (Gems and Partridge 2013, Campisi and di Adda 2007). Studies of the aging can be associated with three basic mechanisms. These are the genetic or environmental changes that influence the rate of aging, the molecular phenotypes which increase with aging and also the altered energy metabolism that shows significant changes in the lifespan and physiological features of selected model organisms (Steinkraus et al. 2008, Luckinbill and Foley 2000). The hallmarks of aging are: genomic instability, telomere attrition, epigenetic alterations, loss of proteostasis, deregulated nutrient-sensing, mitochondrial dysfunction, cellular senescence, stem cell exhaustion, and altered intercellular communication (Lopez-Otin et al. 2013).

However, it is only 30 years since a new era in aging research was inaugurated after the isolation of the first long-lived strains in Caenorhabditis elegans (Klass 1983, Gems and Partridge 2013). Nowadays, aging is subjected to scientific scrutiny based on the ever-expanding knowledge of the molecular and cellular bases of life and disease.

A number of theories, which fall into two main categories, have been proposed in an attempt to explain the process of aging. The first category, aging is caused by the accumulation of damage overtime. Conversely, the latter category of theories suggests various sources and targets of the damage. They are not necessarily mutually exclusive. Rather, aging could vary across different species, and programmed senescence can accelerate the buildup of damage or decrease the capacity repairing (Sergiev et al. 2015). 
Mitochondria are the cellular energy factories that generate ATP via the reaction of hydrocarbons with oxygen. According to the free radical theory of aging, mitochondria generate endogenous ROS which accumulate overtime and create damage to cell (Loeb et al. 2005). In fact, the role of mitochondria in aging process is more complicated than the proposed theories of aging. Multiple changes in mitochondrial function, structure, distribution, and dynamics contribute to aging or agerelated features. Studies in different model organisms have reported that change in mitochondrial function can extend lifespan. Such as, life span can be increased by reduced function of the mitochondria. Mutation or reduced function in nuclear genes encoding electron transport chain (ETC) components in yeast, C. elegans, Drosophila, and mice delay the aging process (Copeland et al. 2009); how the mitochondrial signaling pathway modulating the aging process and the identity of the pathway constituents that transmit these longevity signals remain unknown.

We addressed the question by asking whether manipulation to mitochondrial metabolism genes can extend life span in yeast cell; hence it has been used as a model of organismal and cellular aging for more than 50 years. Kaeberlein (2010) also reported that biology of aging have gained pace over the last century and progress in aging research is now rapid by studying this single cell eukaryote, Saccharomyces cerevisiae.

In recent years, attention has turned toward the understanding of aging in yeast which can be shared with multicellular eukaryotes, including mammals (Longo et al. 2012). There are two primary assays for yeast aging, replicative and chronological (Fontana et al. 2012). Replicative life span (RLS) studies reflect the question how many times one cell can divide. Replicative Life Span (RLS) of a yeast cell refers to the number of daughter cells produced by a mother cell prior to senescence and being determined by counting and removing the buds that they produced, until they size replicate (Fontana et al. 2012). Chronological life span (CLS) is the length of time that a non-dividing yeast cell survives (Steffen et al. 2009, Fontana et al. 2012).

In our present investigation, single deleted mitochondrial metabolism genes of $S$. cerevisiae were used to investigate the role of these genes on lifespan determination. Initially we suspected 12 mitochondrial targeted nuclear genes with reference to different pathways (Table 1) from the source of mitochondrial proteome database (http://www.mitop2.de/). Then those strains, derived from the yeast ORF deletion collection, were screened through the replicative aging assay. Thus, in the current study, we aimed to isolate and identify the metabolism genes that play a role in life span extension.

\section{Yeast strain and culture}

\section{MATERIAL AND METHODS}

The wild-type strain of yeast BY4741 and genetic background isogenic deletion mutants (Table1) were obtained from EUROSCARF and used in this study. The cells were grown in $30^{\circ} \mathrm{C}$ both in solid and liquid YPD broth (1\% yeast extract, 2\% Peptone 2\% Dextrose and 2\% Agar). The concentrations of the yeast cells according to the experimental conditions (optical density) at $600 \mathrm{~nm}$ wave length (OD600) were measured using a spectrophotometer.

Logarithmically growing cells is evaluated as a young population. So the young yeast cells that were used during the experiments were obtained by growing them into a fresh 2\% YPD growth medium for 6 hours at $30{ }^{\circ} \mathrm{C}$. Aged cells were isolated from the culture with the help of a special rotor by centrifugal elutriation system (Beckman Coulter Avanti J-26 XP). Centrifugation and old cell isolation parameter was obtained according to the procedure recommended by the manufacturer. The cells grown at overnight were transferred to a separation chamber of the elutriation system with a pumping rate 40 $\mathrm{ml} / \mathrm{min}$ at $2500 \mathrm{rpm}$. Then the pumping rate was fixed at $30 \mathrm{ml} / \mathrm{min}$ and centrifugation speed at 1000 $\mathrm{rpm}$ to get cells larger than $15 \mu \mathrm{m}$ (senescent cells). The resulting primary (old) cells were stored at $-80^{\circ} \mathrm{C}$. 


\section{Replicative aging assay}

Generalized and conventional protocol was followed from Steffen et al. (2009) with some modifications. Briefly, in this experiment YPD agar plates (1\% yeast extract, 2\% bacto-peptone, $2 \%$ agar and $2 \%$ glucose) were used for culturing yeast cells and for replicative life span analysis. Plates were prepared 2 days before the life span experiment and allowed to dry before beginning the life span assay. Strains were removed from frozen stocks and streaked for single colonies onto YPD agar plates and incubated the cells at $30^{\circ} \mathrm{C}$ for 2 days. Cells were removed from the incubator and patched cells from a single colony onto a fresh YPD plate which was served as the experimental plate used for the replicative life span analysis.

A micro manipulator attached with a Nicon-Eclipse50 microscope was used to dissect daughter cells from the mother cells in every generation; In brief approximately 20 cells from the first patched strain were transferred to a position on the plate distal to the patched cells. The needle of cells was cleaned by repeatedly touching the agar surface, and then a hole was created in the agar by forcing the needle through the agar surface. This hole served as a marker to orient the cells on the plate during daughter cell removal. Once the cells had been arrayed for each patch, the plates were Para filmed and incubated at $30^{\circ} \mathrm{C}$ for approximately 2 hours.

Beginning with the first arrayed cell, we used the needle to detach daughter cells from the mother cell by gently placing the needle on top of the attached cells. The detached daughter cell was placed in the vertical line, replacing the mother cell. All of the mother cells were collected and extra daughter cells were transferred into an area (the graveyard) far from the experimental zone. As the mother cells aged, the cell cycle progression became slower.

The number of daughter cells produced by the mothers were counted and marked in the score sheet and statistical calculation was performed using Wilcoxon Rank-Sum test. The strain that live longer in this experiment was confirmed by repeating the assay twice.

\section{Glycerol spotting assay}

The yeast strains with longer replicative lifespans were grown in YPG (3\% glycerol) and in YPD. In brief, cells were grown in $5 \mathrm{ml}$ liquid YPD at $+30^{\circ} \mathrm{C}$ with shaking. After overnight growth, cells were diluted 1:3 into fresh YPD media and growth of the cells was continued in the same conditions about 3 hours. The OD600 values of the cultures were measured and cells were precipitated then washed with ultrapure water 2 times. Pellets were dissolved in $5 \mathrm{ml}$ ultrapure water. Cultures were diluted to OD600 $=0.2$ and $1 \mathrm{ml}$ of the cultures were transferred into eppendorf tubes. $200 \mu \mathrm{l}$ of the cultures were taken into the 96-well plate randomly. Each culture was diluted seriously in the ratio of 10-fold and range of the concentrations were 2.10-4 to 2.10-1 in an initial volume of $180 \mu 1.5 \mu l$ of each of the cells were spotted onto YPG (1\% yeast extract, $2 \%$ peptone, $2 \%$ agar and 2\% glycerol ) medium and incubated at $+30{ }^{\circ} \mathrm{C}$ incubator over 2 days. After 2 days, spot formations of the cells were examined and plates were photographed.

\section{Study of mitochondrial morphology and the measurement of mitochondrial mass}

Mitochondria specific dye Mitotracker-Red-CMXRos (Thermo Fisher Scientific) was used to stain mitochondria in live cells. Mitochondria were visualized under an Epifluorescence microscope (Olympus BX51) using 100xobjective. Pictures were captured with a CCD camera (Andor technology). Manufacturer provided staining protocol was strictly followed in this study.

Then fluorescence intensity (579/599) of the dye, Mitotracker-Red-CMXRos (Thermo Fisher Scientific), was measured through a flow cytometer (FACS-BD) to quantify mitochondrial density within young and old cell population. Optical density of yeast, OD $600=0.8$ was adjusted for each sample. 
Identification of haploid ppa2d as long living mutant

We used single deleted metabolism genes of $S$. cerevisiae (yeast) to investigate the effects of metabolism on lifespan. Yeast wild type haploid (BY4741) and its isogenic, single deletion mutants that lack 12 mitochondrial targeted nuclear genes with reference to different pathways (Table 1) were screened by Yeast Replicative aging assay.

Table 1. List of the analysed genes with reference to pathways.

\begin{tabular}{llll}
\hline ORF (gene) & Pathway & Function & Subcellular Localization \\
\hline TIM21 & protein sorting & molecular function & mitochondrion \\
MSS2 & protein sorting & molecular function & mitochondrial inner membrane \\
LYS4 & amino acid metabolism & metal ion binding & mitochondrion \\
NFU1 & biogenesis of iron-sulfur cluster & molecular function & mitochondrial matrix \\
SSQ1 & biogenesis of iron-sulfur cluster & nucleotide binding & mitochondrial matrix \\
PPA2 & nucleic acid metabolism & metal ion binding & mitochondrion \\
MRH4 & heme-ubiquinone metabolism & nucleotide binding & mitochondrion \\
REX2 & heme-ubiquinone metabolism & hydrolase activity & mitochondrial matrix \\
PPT2 & lipid metabolism & transferase activity & mitochondrion \\
LSC2 & carbohydrate metabolism & ATP binding & mitochondrial matrix \\
LAT1 & carbohydrate metabolism & transferase activity & mitochondrial matrix \\
KGD1 & carbohydrate metabolism & oxidoreductase activity & mitochondrial matrix \\
\hline
\end{tabular}

Replicative life span refers to the number of daughter cells produced by a mother cell prior to senescence. It is thought to provide a model of aging in mitotically active cells (Steffen et al. 2009). The goal of the replicative life span assay is to determine how many times each mother cell buds. In this study the mother and daughter cells were easily differentiated by using a standard light microscope and physical separation of daughter cells from mother cells was achieved using a manual micromanipulator equipped with a fiber-optic needle.

The average replicative life span of WT strain was measured as 24.5 while ppa2 $\Delta$ was observed as 29.9. In the current investigation ppa $2 \Delta$ showed $22 \%$ life span extension compare to wild type (Fig. 1). Thus the haploid mutant ppa2 $\Delta$ was identified and confirmed as long lived strain. Delaney et al. (2013 identified mitochondrial AFG3 (BY4742) and Caballero et al. (2011) showed mitochondrial translational control gene mutant DSS1 $($ BY4741) as long lived mutant by analyzing Replicative life span. The present study is the first one to identify mitochondrial inorganic pyro phosphatase gene within nucleic acid pathway as longevity gene.

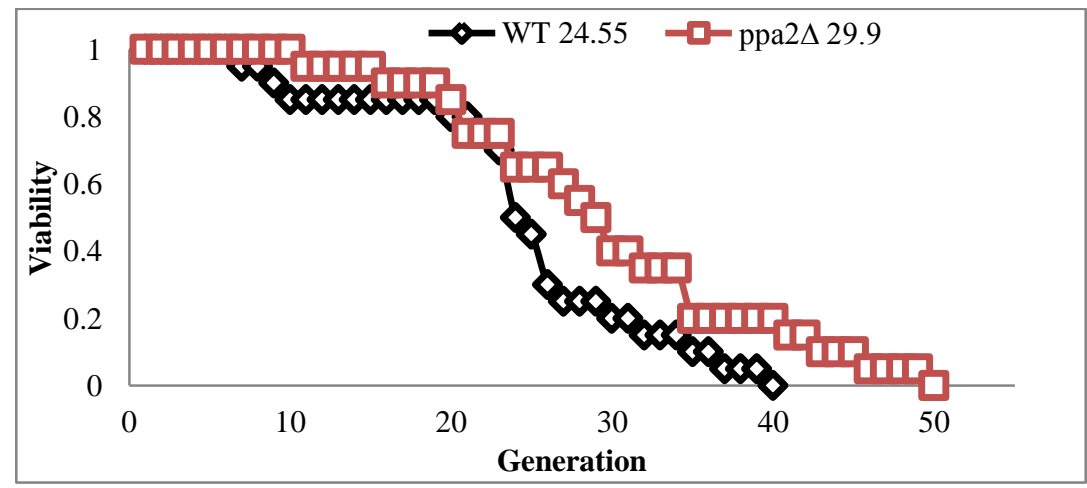

Fig. 1. Yeast Replicative Aging assay: the life span curve of control (WT) and ppa2 $\Delta$ by using Wilcoxon Rank-Sum test. 
Nucleic acid metabolism is important for the maintenance of mitochondrial function. Inorganic pyrophosphates (PPi) formed in metabolic activities and their hydrolysis necessary to sustain these metabolic activities. PPA2 gene encodes yeast mitochondrial inorganic pyro phosphatase that hydrolysis PPi formed during the mitochondrial DNA synthesis (Lundin et al. 1991).

Among 12 gene deletion mutants, only ppa2 $\Delta$ was ensured as long lived strains using haploid cells (BY4741) which implies that the deletion of that gene has regulatory role for life span determination. When we screened the other genes in the pathways, they did not show extended life span that suggests that the effect of deletion on life span is solely gene specific.

\section{Mitochondrial morphology, density and distribution pattern of the long living mutant}

As mitochondrial inorganic pyro phosphatase PPA2 is required for mitochondrial DNA synthesis and mitochondrial function (Lundin et al. 1991), so disruption of the gene was suspected to affect mitochondrial morphology and functions. In the present study, mitochondrial morphology and distribution were investigated through fluorescence microscope (Olympus BX51). Mitochondrial staining dye Mitotracker Red CmxRos was used to visualize mitochondria in vivo. WT cell exhibited serpentine nature of mitochondrial chain network as expected while mitochondria aggregated and formed colonies within the all long lived cells (Fig. 2). Both young and old long lived cells have similar fashion of aggregation.

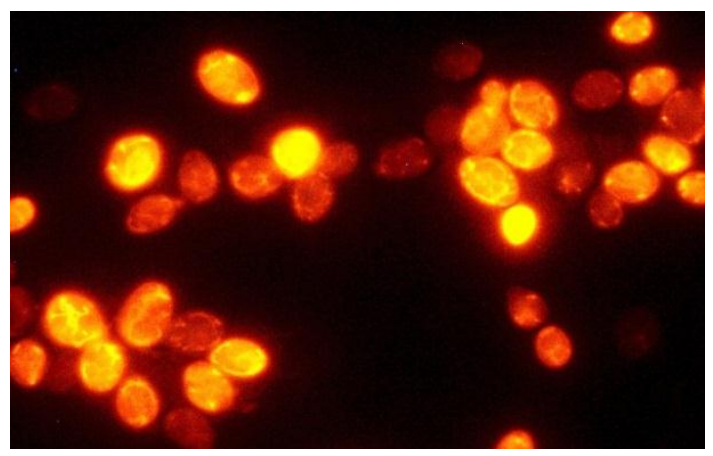

a

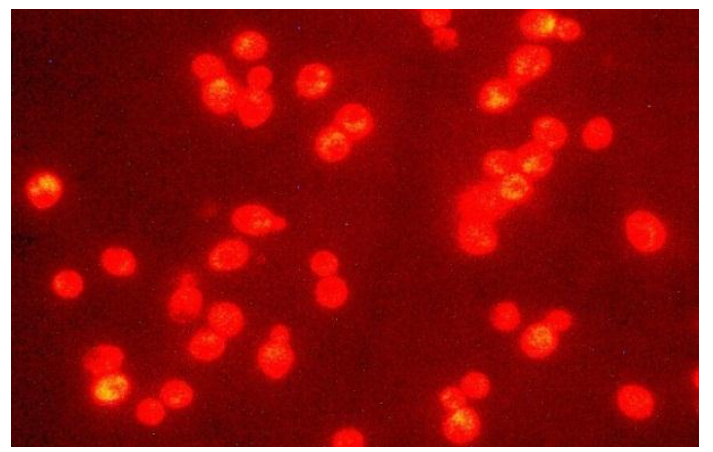

b

Fig. 2. Fluorescence microscopic analysis was performed for both young and old cells. a. WT young and old cell comprised mitochondrial network; b. Aggregated and colonized pattern of mutant's (ppa2 $\Delta$ ) mitochondria in the cytoplasm. Both young and old ppa $2 \Delta$ had similar incidence.

Mitochondrial density was measured by using the same dye that was used for visualization. After staining the mitochondria, intensity was measured through a flow cytometer (FACS BD). Long lived cell in this study showed almost equal amount of mitochondrial mass compared to wild type (Fig. 3) which suggests that mitochondria were not destroyed after breaking down of network and in aggregated state.

In the present investigation, long lived cell's mitochondria were seen as aggregated and colonized instead of chain networking form while the content of mitochondria remained intact in the course of young to old stage (Fig. 2). This finding suggests that long lived cells had developed an alternate morphology. The mechanism of breaking down of mitochondrial network was not investigated in this current study, but Chistiakov et al. (2014) reported that the regulation of mitochondrial architecture is mediated by fusion (join)/fission (separation) events and mitochondria are selectively removed by mitophagy that degrades imperfectly functional mitochondria.

It has been shown that older mother cells have a tendency to segregate dysfunctional mitochondria to their daughters (Jazwinski 2004, Veatch et al. 2009, Lanza and Nair 2010). Similarly all the young cells 
in this study were shown to have almost equal amount of mitochondrial mass compared to old one (Fig. 3 ) which suggests that mitochondria were not destroyed, rather it might function in another way.

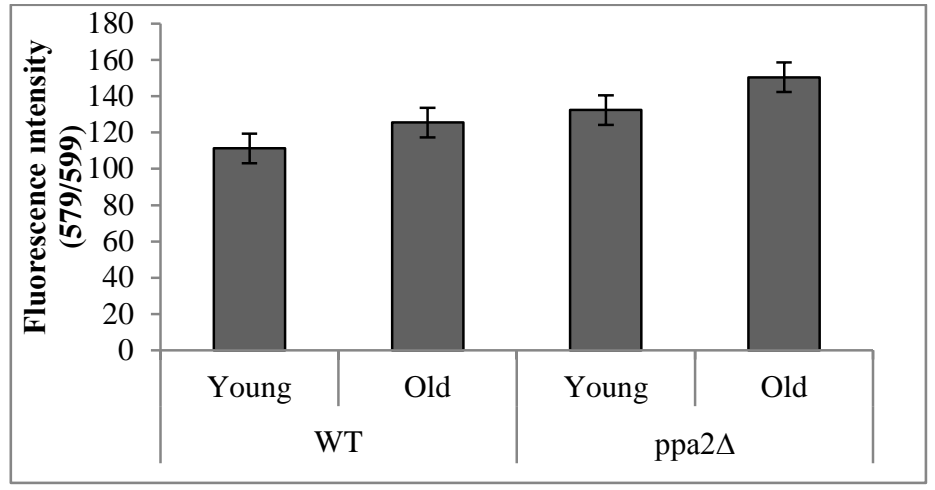

Fig. 3. Mitochondrial density was quantified by measuring the fluorescence intensity (579-excitation/599-emission) of the mitochondria specific dye, MitotrackerRed-CMXRos, through a flow cytometer (FACS-BD).

Katharine et al. (2012) reported that the mitochondrial fission-fusion cycle plays a role in mitophagy, since deletion of DNM1, in fact this gene is required for fission, attenuates mitophagy without entirely eliminating it (Kanki et al. 2009). Interestingly, deletion of this gene extends yeast replicative lifespan (Scheckhuber et al. 2007). Another possible explanation was viewed from the yeast genome data base (http://www.yeastgenome.org/) where the gene PPA2 was shown to be interacted genetically with DNM1, MDM, and ARD1complex that play strong roles in mitochondrial localization, fission, fusion and early mitophagy (http://www.yeastgenome.org). Taken together these data, it is assumed that the deletion of PPA2 gene may lead to loss of the interactive network with the regulatory genes that may lead to mitochondrial aggregation. And the colonized pattern of aggregation may be a protective morphology against mitophagy. Further investigation is required to verify this hypothesis.

\section{The respiration and proliferation status of ppa2s}

To determine respiratory phenotype of this mutant, we grew the cells on $3 \%$ glycerol containing YPG medium. Since the enzyme inorganic pyro phosphatase is required for mitochondrial DNA metabolic activities through oxidative metabolism, we tested growth defects on nonfermentable carbon source. In this experiment, ppa2 $\Delta$ was not able to utilize glycerol as the carbon source to derive energy. Thus, this long lived cell is respiratory incompetent (Fig. 4). We do hypothesize that the ppa2 gene may have a unique role in the maintenance of mitochondrial morphology and function.

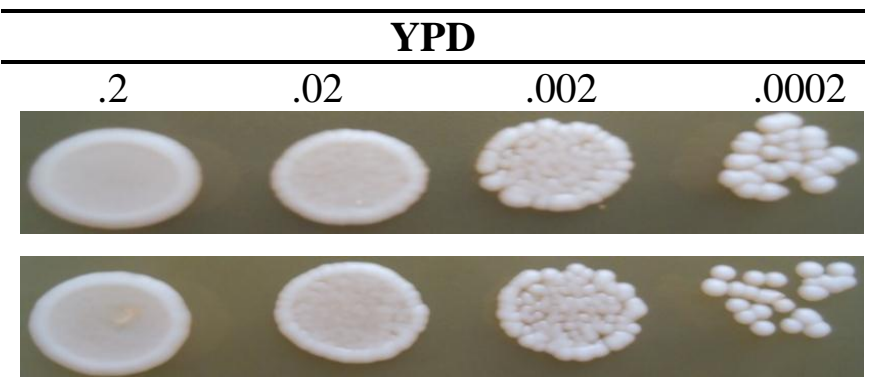

a

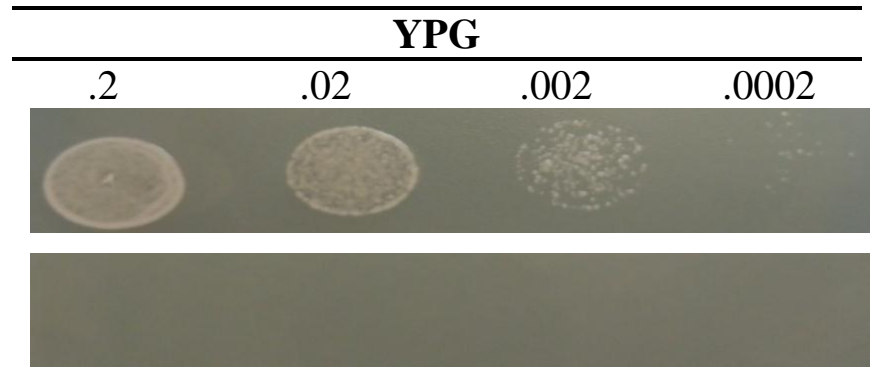

b

Fig. 4. Colony morphology: a. both WT and ppa2 $\Delta$ cells are similar when they were grown in YPD media; b. long living mutant cell could not grow in glycerol containing media. 
The most common spontaneous events that result in altered mitochondrial function in budding yeast are either mtDNA rearrangements $\left(\rho^{-}\right)$, or total loss of the mtDNA $(\rho 0)$, which prevent the production of mitochondria-encoded proteins. Saccharomyces cerevisiae rho0 cells (petite), which lack mtDNA, were defective in G1- to S-phase progression and thus, the growth defect in rho0 cells is caused by loss of DNA within mitochondria (Crider et al. 2012).

A slow growth rate in petite culture could result from a high frequency of cell death, cell-cycle arrest, and/or a slower cell cycle. Though mitochondrial DNA of the long lived mutant was not quantified, growth and proliferation status was observed in the current study. Our studied long lived young cell rendered the same colony shape compared to wild type when they are grown in YPD (Fig. 4). The proliferation rate was viewed through analyzing the growth curve in YPD (Fig. 5) and we did not find any growth defect which implies that this long lived mutant behave differently from petite characteristics.

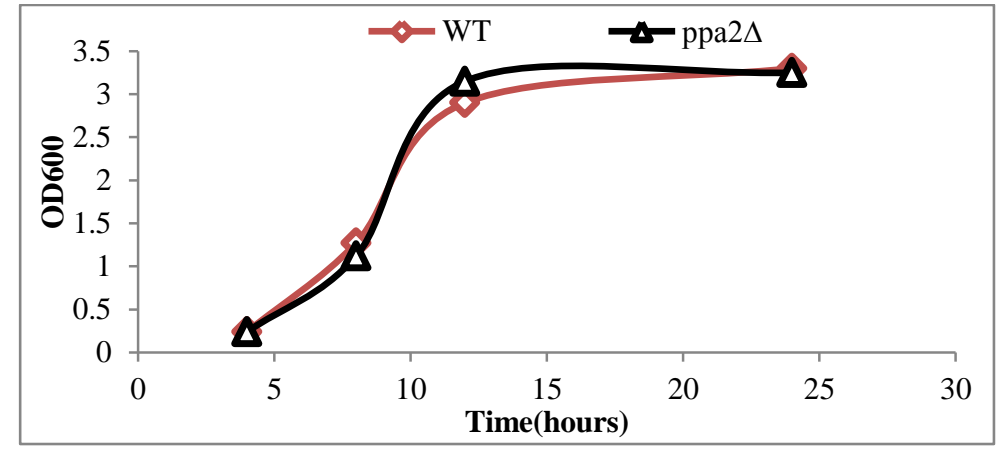

Fig. 5. The growth curve of ppa2 $\Delta$ in YPD media. (Optical density $600 \mathrm{~nm}$ was measured in a spectrophotometer).

Thus the current study reveals that PPA2 gene knocks out generates an alternative phenotype on mitochondrial morphology and function that harbor life span extension. Further biochemical and genetic investigations are highly required to find out the mechanism of this longevity pathway.

\section{ACKNOWLEDGEMENTS}

This work was partially supported by the Scientific and Technological Research Council of Turkey. Authors are also grateful to Professor Alper (Virology lab, Department of Molecular Biology and Genetics, Izmir Institute of technology, Turkey) for providing fluorescence microscopic facilities.

\section{REFERENCES}

Caballero, A., A. Ugidos, B. Liu, D. Oling and T. Nystrom. 2011. Absence of miochondrial translation control proteins extends life span by activating sirtuin dependent silencing. Mol. Cell. 42(3): 390-400.

Campisi, J. and F. F. di Adda. 2007. Cellular senescence: when bad things happen to good cells. Nat. Rev. Mol. Cell Biol. 8: 729-740.

Chistiakov, D. A., A. I. Sobenin, V. V. Revin, A. N. Orekhov and Y. V. Bobryshev. 2014. Mitochondrial aging and age related dysfunction of mitochondria. BioMed. Res. Int. 20(14): 1-7.

Copeland, J. M., J. Cho, J. H. Hur and D. W. Walker. 2009. Extension of Drosophila Life Span by RNAi of the Mitochondrial Respiratory Chain. Current Biol. 54: 456-62.

Crider, D. G., L. J. Garcia-Rodriquez, P. Srivastava, L. Peraza-Reyes and L. A. Pon. 2012. Rad53 is essential for mitochondrial DNA inheritence checkpoint regulating G1 to S progression. J. Cell Biol. 198(5): 793-798. 
Delaney, J, R., U. Ahmed, A. Chou and M. Kaeberlein. 2013. Stress profiling of longevity mutants identifies Afg3 as a mitochondrial determinant of cytoplasmic mRNA translation and aging. Aging cell. 12(1): 156166.

Farooqui, T. and A. A. Farooqui. 2008. Aging: an important factor of the pathogenesis of neurodegenerative diseases. Mech. Aging Dev. 130(4): 203-215.

Fontana, L., L. Partridge and D. V. Longo. 2012. Extending healthy life span from yeast to human. Science. 328: 321-326.

Gems, D. and L. Partridge. 2013. Genetics of longevity in model organisms: debates and paradigm shifts. Annu. Rev. Physiol. 75: 621-644.

Jazwinski, S. M. 2004. Yeast longevity and aging-the mitochondrial connection. Mech. Ageing Dev. 126: 243248.

Kaeberlein, M. 2010. Lessons on longevity from budding yeast. Nature. 465(25): 513-519.

Kanki, T., K. Wang, M. Baba, C. R. Bartholomew, M. A. Lynch-Day, Z. Du, J. Geng, K. Mao, Z. Yang, W. L. Yen and D. J. Klionsky. 2009. A genomic screen for yeast mutants defective in selective mitochondrial autophagy. Mol. Biol. Cell. 20: 4730-4738.

Katharine, B., L. Yufi and D. Chen. 2012. Aging: The Mitochondrial Connection. J. Clin. Exp. Pathol. 4(3): 432438.

Klass, M. R. 1983. A method for the isolation of longevity mutants in the nematode Caenorhabditis elegans and initial results. Mech. Ageing Dev. 22(3-4): 279-286.

Lanza, I. R. and K. S. Nair. 2010. Mitochondrial function as a determinant of life span. Eur. J. Physiol. 459(2): 277-289.

Loeb, L. A., D. C. Wallace and G. M. Martin. 2005. The mitochondrial theory of aging and its relationship to reactive oxygen species damage and somatic mtDNA mutations. Proc. Natl. Acad. Sci USA. 102(52): 1876918770.

Longo, V. D., G. S. Shadel, M. Kaeberlein and B. Kennedy. 2012. Replicative and Chronological Aging in Saccharomyces cerevisiae. Cell Metab. 16(1): 18-31.

Lopez-Otin, C., M. A. Blasco, L. Partridge, M. Serrano and G. Kroemer. 2013. The hall marks of aging. Cell. 153(6): 1194-1217.

Luckinbill, L. S. and P. Foley. 2000. The role of metabolism in ageing. J. Am. Aging Assoc. 23(2): 85-93.

Lundin, M., H. Baltscheffsky and H. Ronne. 1991. Yeast PPA2 gene encodes a mitochondrial inorganic pyrophosphatase that is essential for mitochondrial function. J. Biol. Chem. 266(19): 12168-12172.

Scheckhuber, C. Q., N. Erjavec, A. Tinazli, A. Hamann, T. Nystrom and D. Osiewacz. 2007. Reducing mitochondrial fission results in increased life span and fitness of two fungal ageing models. Nat. Cell. Biol. 9(1): 99-105.

Sergiev, P. V., O. A. Dontsova and G. V. Berezkin. 2015. Theories of Aging: An Ever-Evolving Field. Acta Naturae. 7(1): 9-18.

Steffen, K. K., B. K. Kennedy and M. Kaeberlein. 2009. Measuring replicative life span in budding yeast. J. Vis. Exp. 28: 566-572.

Steinkraus, K. A., M. Kaeberlein and B. K. Kennedy. 2008. Replicative aging in yeast: the means to the end. Annu. Rev. Cell Dev. Biol. 24: 29-54.

Veatch, J. R., M. A. McMurray, Z. W. Nelson and D. E. Gottschling. 2009. Mitochondrial dysfunction leads to nuclear genome instability: A link through iron sulfur clusters. Cell. 137(7): 1247-1258. 